\title{
Monitoring thiopurine metabolites in inflammatory bowel disease
}

\author{
Yago González-Lama, ${ }^{1}$ Javier P Gisbert ${ }^{2}$
}

\begin{abstract}
${ }^{1}$ Gastroenterology and Hepatology Department, Puerta de Hierro University Hospital, Majadahonda, Madrid, Spain ${ }^{2}$ Gastroenterology Unit, Hospital Universitario de La Princesa and Instituto de Investigación Sanitaria Princesa (IIS-IP), Centro de Investigación Biomédica en Red de Enfermedades Hepáticas y Digestivas (CIBEREHD), Madrid, Madrid, Spain
\end{abstract}

\section{Correspondence to} Dr Javier P Gisbert, Playa de Mojácar 29, Urb. Bonanza, Boadilla del Monte, Madrid 28669, Spain; javier.p.gisbert@ gmail.com

Received 30 December 2015 Revised 9 March 2016 Accepted 16 March 2016 Published Online First 7 April 2016

\section{CrossMark}

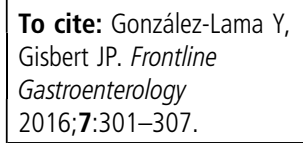

\begin{abstract}
Thiopurines (azathioprine and mercaptopurine) are one of the immunosuppressive mainstays for the treatment of inflammatory bowel disease. In spite of its widespread use, thiopurine metabolism is still not fully understood, and a significant proportion of patients suffer toxicity or lack of efficacy. Different enzymatic pathways with individual variations constitute a pharmacogenetic model that seems to be suitable for monitoring and therapeutic intervention. This review is focused on current concepts and recent research that may help clinicians to rationally optimise thiopurine treatment in patients with inflammatory bowel disease.
\end{abstract}

\section{INTRODUCTION}

Inflammatory bowel disease (IBD) is a chronic and disabling inflammatory disorder that is caused by an incompletely known immunological dysregulation in a genetically predisposed patient. The main two conditions comprised in the IBD spectrum are Crohn's disease (CD) and ulcerative colitis (UC).

One of the bases of the therapeutic approach for IBD is the use of immunomodulatory drugs, in an attempt to correct the immunological disorder that causes the disease. Thiopurines, azathioprine (AZA) or mercaptopurine (MP), have been widely used as steroid-sparing agents and are indicated to maintain remission in both CD and UC, although their mechanism of action is still not fully understood. In spite of their widespread use, between 30 and $50 \%$ of the patients have to discontinue thiopurines because of adverse events or lack of efficacy. ${ }^{1-8}$ Since efficacy and toxicity of thiopurines are thought to be related to individual variations of thiopurine metabolism, research has been focused on understanding, monitoring or even modifying the metabolic pathways of thiopurines in each particular patient. 910

This article is focused on the current concepts of thiopurine metabolism and on the most recent studies that may help clinicians to optimise thiopurine treatment in patients with IBD.

\section{THIOPURINE METABOLIC PATHWAY}

The first step of AZA metabolism is its conversion into MP. This process occurs in the liver, and up to $90 \%$ of the process is non-enzymatic, although at least a small proportion of this conversion can be related to the enzyme glutathione S-transferase (GST). ${ }^{10}{ }^{11}$ As a matter of fact, deletion of GST-M1 (which determines reduced enzymatic activity) has been recently related to reduced active metabolite concentrations and reduced sensitivity to AZA in young patients. ${ }^{12}$

Both AZA and MP are prodrugs, which require intracellular activation by a complex multienzymatic process involving three competing pathways via three different critical enzymes (figure 1): hypoxanthine phosphoribosyl transferase (HPRT), thiopurine methyltransferase (TPMT) and xanthine oxidase (XO). HPRT converts AZA/MP into 6-thioinosine monophosphate (6TIMP), which further metabolises into the active cytotoxic metabolite 6thioguanine nucleotide (6TGN) through a multienzymatic pathway that includes also TPMT, inosine monophosphate dehydrogenase (IMPDH) and guanosine monophosphate synthetase (GMPS). IMPDH directly converts 6TIMP to 6TGN, avoiding the intermediate 6-methylmercaptopurine ribonucleotide (6MMPR), which may have hepatotoxic effects and probably limits thiopurine phosphorylation. XO metabolises an important part of MP into the inactive agent 6-thiouric acid, and TPMT catalyses two reactions resulting in 


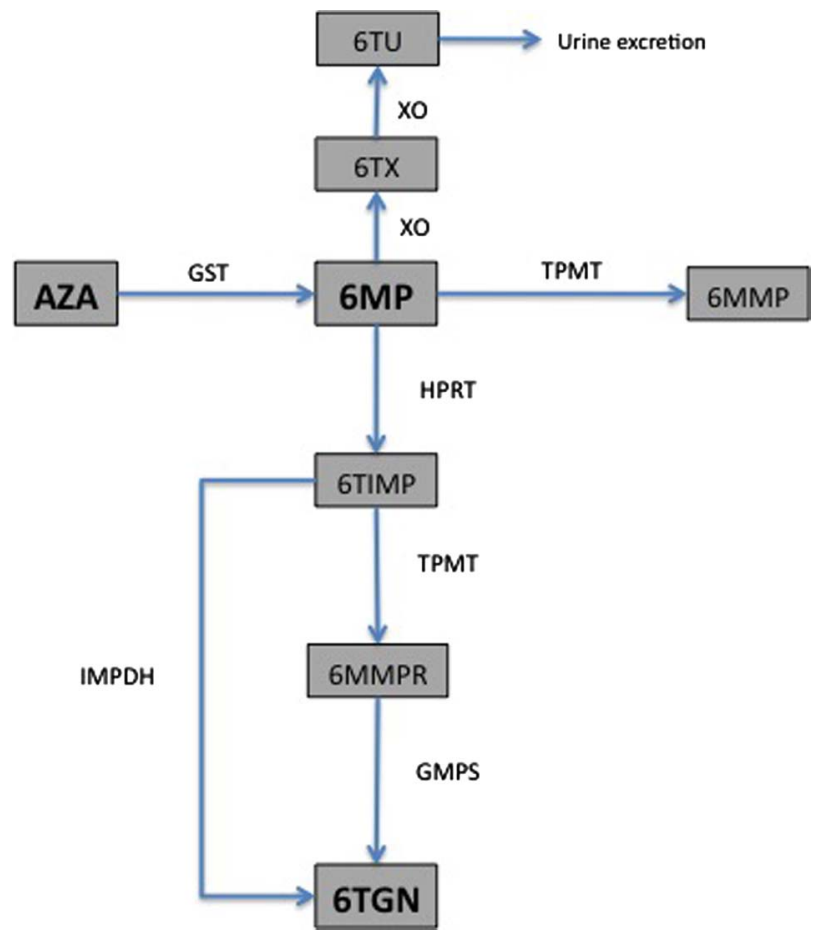

Figure 1 Thiopurine metabolic pathway.

the formation of 6-methylmercaptopurine (6MMP) and 6MMPR, which may be responsible for some toxic effects like hepatotoxicity. ${ }^{9} 11$ 13-18

6TGN has immune modifier activity and is considered the main therapeutic metabolite of thiopurines, but may lead to myelosuppression. It is a purine antagonist that inserts within the DNA of leucocytes, and is therefore responsible for inhibiting its DNA synthesis and subsequent proliferation of T lymphocytes. ${ }^{11} 161819$ Besides that, some other immunomodulating properties that may play a role in downregulating factors involved in intestinal inflammation have been associated to 6TGN: it has been found to inhibit tumour necrosis factor related apoptosis ligand, $\alpha 4$-integrin in activated $\mathrm{T}$ lymphocytes or the expression of the Rac1 protein (implicated in T-cell proliferation), leading all of these to immunosuppression through a wide spectrum of immunosuppressive roles. ${ }^{11} 2021$

TPMT is the principal enzyme in the regulation of thiopurine metabolism. The TPMT gene has an autosomal codominant inheritance; occasional genetic polymorphism has been described regarding the TPMT activity, which results in a trimodal distribution; those patients heterozygous or homozygous for the 'low activity' mutation gene may have a special susceptibility for myelotoxicity with thiopurine therapy. Approximately $90 \%$ of the Caucasian population is homozygous for the wild-type TPMT gene and have normal or high activity of this enzyme; it is a commonly held belief that this characteristic provides a safer condition regarding the intake of thiopurines. Approximately $10 \%$ and $0.5 \%$ of this population are heterozygous for intermediate enzyme activity and homozygous for the low enzyme activity, respectively. Interestingly, allelic frequency patterns also vary among ethnic groups. ${ }^{22}$

It is believed that low TPMT activity results in high 6TGN levels and higher associated risk of leucopenia. Therefore, patients with low TPMT activity should not receive thiopurines; if AZA or MP are considered in this kind of patients, low doses and tight hematological monitoring should be warranted. ${ }^{23}{ }^{24}$ On the other hand, high TPMT activity results in less 6TGN levels, and therefore less therapeutic efficacy, but more 6MMP, which may cause hepatotoxicity. Moreover, higher TPMT activity $(>14 \mathrm{U} / \mathrm{mL}$ red blood cells $(\mathrm{RBC})$ ) preferentially produces 6MMP instead of 6TGN, and it could explain the lack of clinical response and increased risk of hepatotoxicity in these patients. $151825-29$

\section{MONITORING THIOPURINE METABOLITES}

Traditional approach to AZA dosing is weight-based, and is usually established at approximately $2.5 \mathrm{mg} / \mathrm{kg} /$ day. Regarding MP, it is usually dosed at half of AZA, which means $1-1.5 \mathrm{mg} / \mathrm{kg} /$ day, since $\mathrm{MP}$ is approximately half of AZA in terms of molecular weight, and almost $90 \%$ of AZA is finally converted to MP. However, individual variations in thiopurine metabolism are the causes of interindividual differences in both therapeutic benefit and adverse events profile. These differences could be higher than $50 \%$, in terms of therapeutic response. ${ }^{4} 915^{27} 29-33$ Recent research in monitoring serum levels of active metabolites of thiopurines may help clinicians to optimise effectiveness and safety of thiopurine treatment.

Despite the widespread use of thiopurines and the growing knowledge about their interindividual variations in metabolism, monitoring of thiopurine metabolites is not widely used: at least two recently published surveys reveal that determination of TPMT activity (genotype or phenotype), 6TGN or 6MMP levels is used by a relatively low proportion of IBD gastroenterologist, the availability or reimbursement issues being the most relevant obstacles. ${ }^{34} 35$

\section{Efficacy and safety of thiopurines}

Even though 6TGN is the active metabolite of thiopurine drugs, high 6TGN levels can also be the cause of myelosuppression. Clinical benefit of thiopurine drugs has been related with serum 6TGN levels between 235 and $450 \mathrm{pmol} / 8 \times 10^{8} \mathrm{RBC}$, although the existence of a specific and truly reliable 6TGN cut-off level still remains controversial. ${ }^{26} 3637$ This association between 6TGN serum levels and clinical benefit has been shown with clinical response and with endoscopic improvement of CD mucosal lesions. ${ }^{38}$

Although some previous studies suggested that the correlation between 6TGN serum levels and weightbased thiopurine dosing might be poor, recent data have shown good positive correlation between oral 
dosage of AZA and 6TGN serum levels in paediatric population. ${ }^{33}{ }^{39-41}$ Therefore, adjusting AZA dosing according to 6TGN serum levels can be a useful tool leading to improved outcomes at least in cases of inefficacy or adverse events, as it has been recently shown in a retrospectively observational study focused on the clinical usefulness of thiopurine metabolites monitoring in South Australia. ${ }^{42}$ In spite of all of that, a recent multicentre, double-blind randomised controlled trial failed to demonstrate superiority of individualised AZA dosing based on 6TGN serum levels over classical weight-based dosage approach of AZA in patients with IBD, ${ }^{43}$ although the size of the population studied was probably underpowered and the investigators reported inability to achieve the target 6TGN concentrations in the individualised arm. ${ }^{44}$

There have been many attempts to identify haematological indices as surrogate markers for therapeutic success of thiopurine, which is a useful tool to optimise thiopurines dose in clinical practice. These strategies include monitoring changes in the red cell mean corpuscular volume (MCV) or induction of leucopenia. Even though leucopenia is a well-recognised effect of thiopurines and closely related to their therapeutic mechanism, its association with therapeutic efficacy has yet to be determined. In spite of recent research, correlation between 6TGN serum levels and lymphopenia or leucopenia still remains uncertain, although a prospective observational study has recently shown that $\mathrm{MCV}>101 \mathrm{fL}$ reliably excluded subtherapeutic 6 TGN levels. $^{33} 4145-47$

There are some other determinations that can be useful to understand the thiopurine metabolism of an individual patient. Genetic polymorphisms in TPMT have been related to interindividual differences in efficacy and safety. TPMT activity has been found to be inversely related to the clinical response to thiopurines, while a meta-analysis found that low TPMT activity was related to myelotoxicity but not hepatotoxicity or pancreatitis. ${ }^{48}$ Established skewed metabolism of thiopurines that preferentially produces 6MMP rather than $6 \mathrm{TGN}$ is probably the major risk factor for thiopurine failure (lack of efficacy or adverse events) in patients with IBD. Identifying those patients may be a very useful prognostic tool that may help clinicians to provide adequate therapeutic approach to each individual patient. ${ }^{49}{ }^{50}$ High 6MMP levels (>5700 pmol $/ 8 \times 10^{8} \mathrm{RBC}$ ) are associated with adverse events, and a retrospective study found that lack of response to AZA or MP was associated to a high 6MMP/6TGN ratio. 5951

Many dosing strategies have been proposed according to the TPMT activity, although no one is evidencebased. The classic approach stands for starting thiopurines at low doses and gradually titrating in an attempt to avoid adverse events; this strategy can be safe but long-lasting. TPMT determination may be useful to select those patients with high TPMT activity to receive weight-based full dose from the start, in a safe and also faster and cost-effective way. Appropriate dosing for patients with intermediate or low dose still remains controversial. ${ }^{1} 41152-54$

However, the thiopurine metabolism is still not fully understood, and some recent evidence suggests that there should be alternative metabolic pathways for preferentially producing 6MMP beyond high TPMT activity. ${ }^{55}$ Thus, some genetic variants of TPMT related to bone marrow toxicity have been recently identified; in patients carrying any of those TPMT variants, dose reduction may avoid haematological events. ${ }^{56-58}$

\section{Adherence to thiopurines}

Determination of 6TGN serum levels has been employed also to successfully identify non-adherent or underdosed patients. This is especially relevant in those non-responding patients: low levels of 6TGN and $6 \mathrm{MMP}$ may lead to strength measures to improve adherence or to increase the dose of thiopurines. Moreover, some evidence suggests that medical supervision based on the routine determination of thiopurine metabolites may improve adherence to therapy, and therefore result in better outcomes. ${ }^{59} 60$

\section{Thiopurine-combined treatments}

Combination of AZA or MP with aminosalicylates is very common in clinical practice. ${ }^{61}$ Many studies have found that aminosalicylates increase 6TGN levels in a majority of the patients, although whether or not this increase has clinical significance still remains controversial. In a small prospective study carried in Asian patients, the myelotoxicity rate was $47 \%$ in the combination group, while it was only $16 \%$ in the thiopurine monotherapy group; on the other hand, a previously reported cross-sectional study including 14545 Spanish patients showed that combination with aminosalicylates was associated with a slight increase of 6TGN levels, which was statistically significant but not clinically relevant. ${ }^{25}$

Therefore, if the combination of thiopurines with aminosalicylates is associated with higher probabilities of therapeutic success, higher risk of myelotoxicity or deserve a pre-emptive lower AZA dosing with a tight metabolite monitoring still remains unclear. ${ }^{11} 2562 \quad 63$ Probably, the answer depends on the genetic characteristic of the population considered. As a matter of fact, a recently published pilot study found that genetic polymorphisms of $\mathrm{N}$-acetyl transferase could affect 6TGN levels in patients treated with thiopurines and aminosalicylates and could therefore influence the toxicity and efficacy of these drugs. ${ }^{64}$

Combination of thiopurines with anti-tumor necrosis factor (TNF) agents is also not uncommon. A randomised controlled trial showed that early initiation of combination therapy with AZA and infliximab was associated with better outcomes, including mucosal 
healing, when compared with AZA or infliximab alone. ${ }^{65}$ It also has been proposed that combo therapy may reduce immunogenicity to biologics and improve therapeutic outcomes. On the other hand, at least part of the added efficacy of the combination therapy is owing to increased trough levels of the biologics. ${ }^{65} 66$

Even though combining anti-TNF agents and thiopurines seems to be beneficial in terms of efficacy, some safety concerns about combination therapy lead clinicians to discontinue thiopurines after a period of time, but this strategy may have implications in longterm efficacy of the drug. ${ }^{6768}$ A recent cross-sectional study showed that $6 \mathrm{TGN}$ levels of only $125 \mathrm{pmol} /$ $8 \times 10^{8} \mathrm{RBC}$ were enough to predict higher infliximab levels and the absence of antibodies to Infliximab (ATI), which is relevant since it points out the possibility of decreasing dose of AZA as an alternative to thiopurine withdrawal. ${ }^{66}$

In addition, combination therapy may have other benefits since some evidence suggests that direct drug interaction between AZA and infliximab may result in higher 6TGN levels. ${ }^{69} 70$ However, a recently published small prospective study found no pharmacokinetic drug interaction between thiopurines and adalimumab. ${ }^{71}$

\section{STRATEGIES TO OPTIMISE THIOPURINE TREATMENT BASED ON METABOLITES DETERMINATION}

As previously stated, skewed metabolism that preferentially produces $6 \mathrm{MMP}$ instead of $6 \mathrm{TGN}$ seems to be the major cause of thiopurine failure. Dose escalation to achieve therapeutic 6TGN levels may lead to high $6 \mathrm{MMP}$ levels. Increased $6 \mathrm{MMP} / 6 \mathrm{TGN}$ ratio is associated with poor therapeutic response and side effects that comprise headache, malaise, nausea, myalgia and hepatotoxicity.

A recently published retrospective study showed that dividing total daily dose of AZA or MP in thiopurine-intolerant patients with high 6MMP levels led to a significant reduction of 6MMP serum levels while maintaining $6 \mathrm{TGN}$ above therapeutic levels, with the resolution of the 6MMP-related adverse events. ${ }^{72}$ These findings suggest that this dose-splitting strategy may lead to a change in the metabolism profile and can be considered as an alternative in this group of patients. Tight metabolite monitoring should be considered to avoid toxicity or underdose of thiopurines. ${ }^{11}$

Adding allopurinol to thiopurine therapy is another proposed alternative for those preferential 6MMP metabolisers since it has been shown to improve therapeutic $6 \mathrm{TGN}$ levels and decrease serum concentrations of toxic 6MMP metabolite. ${ }^{73-75}$ The precise mechanism by which allopurinol is useful in shifting metabolites towards 6TGN production remains unclear, although some alternatives have been pointed out: first, direct inhibition of XO would result in shifting conversion to the remaining pathways, and subsequently to increased serum levels of active 6TGN metabolites; second, allopurinol supplementation would inhibit TPMT through the production of 6-thioxanthine and finally, the last proposed mechanism is an increased activity of HPRT enzyme towards the active pathway. ${ }^{117677}$

Classic management of this combination of thiopurine plus allopurinol usually includes reducing thiopurines at least by $50 \%$ and adding $100 \mathrm{mg}$ of allopurinol,

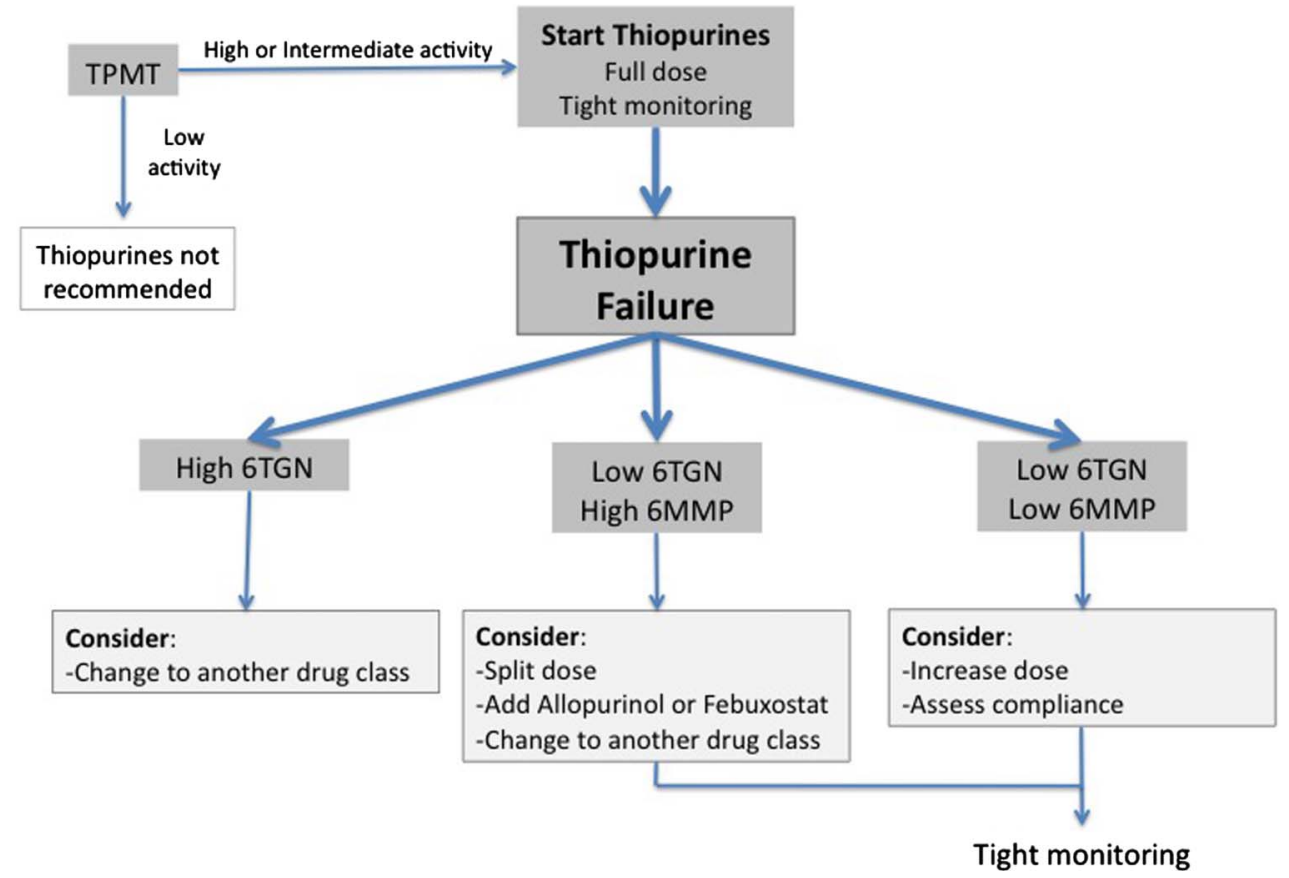

Figure 2 Proposed management of thiopurines treatment. 6MMP, 6-methylmercaptopurine; 6TGN, 6-thioguanine nucleotide; TPMT, thiopurine methyltransferase. 
although recent research suggests that lower doses could also be useful and safer. ${ }^{11} 78$ Tight metabolite and haematological monitoring is mandatory, and usually complete cell count at weekly basis during the first month and every other week during the second month is recommended. ${ }^{11}$

Finally, a novel XO inhibitor named febuxostat has shown, in a case study, to be useful in shifting thiopurine metabolism by preventing $6 \mathrm{MMP}$ production and increasing $6 \mathrm{TGN}$ levels. ${ }^{79}$

\section{CONCLUSIONS}

Thiopurine metabolism is complex and still not fully understood, but it represents a pharmacogenetic model suitable for therapeutic intervention. Current knowledge of different metabolic pathways allows clinicians to monitor and optimise the thiopurine metabolism of an individual patient. An algorithm with the proposed management of thiopurine treatment according to metabolite determination is provided (figure 2).

Determination of TPMT activity may help to identify those patients at higher risk of developing toxicity, and monitoring thiopurine metabolites may help clinicians to optimise the dose of AZA or MP at least in cases of lack of efficacy, suspect of unsatisfactory compliance or in the presence of adverse events. However, there is not enough data to support routine and systematic determination of thiopurine metabolites for every patient, which would be expensive and not always available. Combination strategies with other drugs may result in better outcomes, but require expertise and tight monitoring.

Twitter Follow Yago González-Lama at @YagoGlezLama

Contributors Both authors have substantially contributed to the manuscript. YG-L: conception and design of the article, writing the manuscript, reviewed the draft and submission. JPG: conception and design of the article, reviewed the draft for important intellectual content and final approval of the version to be submitted.

Competing interests None declared.

Provenance and peer review Commissioned; internally peer reviewed.

\section{REFERENCES}

1 Chande N, Tsoulis DJ, MacDonald JK. Azathioprine or 6-mercaptopurine for induction of remission in Crohn's disease. Cochrane Database Syst Rev 2013;4:Cd000545.

2 Gordon M, Taylor K, Akobeng AK, et al. Azathioprine and 6-mercaptopurine for maintenance of surgically-induced remission in Crohn's disease. Cochrane Database Syst Rev 2014;8:Cd010233.

3 Prefontaine E, Sutherland LR, Macdonald JK, et al. Azathioprine or 6-mercaptopurine for maintenance of remission in Crohn's disease. Cochrane Database Syst Rev 2009 (1): Cd000067.

4 Timmer A, McDonald JW, Tsoulis DJ, et al. Azathioprine and 6-mercaptopurine for maintenance of remission in ulcerative colitis. Cochrane Database Syst Rev 2012;9:Cd000478.
5 Jharap B, Seinen ML, de Boer NK, et al. Thiopurine therapy in inflammatory bowel disease patients: analyses of two 8-year intercept cohorts. Inflamm Bowel Dis 2010;16:1541-9.

6 Magro F, Santos-Antunes J, Vilas-Boas F, et al. Crohn's disease outcome in patients under azathioprine: a tertiary referral center experience. J Crohns Colitis 2014;8:617-25.

7 Chaparro M, Ordás I, Cabré E, et al. Safety of thiopurine therapy in inflammatory bowel disease: long-term follow-up study of 3931 patients. Inflamm Bowel Dis 2013;19:1404-10.

8 Gisbert JP, Linares PM, McNicholl AG, et al. Meta-analysis: the efficacy of azathioprine and mercaptopurine in ulcerative colitis. Aliment Pharmacol Ther 2009;30:126-37.

9 Dubinsky MC, Lamothe S, Yang HY, et al. Pharmacogenomics and metabolite measurement for 6-mercaptopurine therapy in inflammatory bowel disease. Gastroenterology 2000;118:705-13.

10 Sahasranaman S, Howard D, Roy S. Clinical pharmacology and pharmacogenetics of thiopurines. Eur J Clin Pharmacol 2008;64:753-67.

11 Amin J, Huang B, Yoon J, et al. Update 2014: advances to optimize 6-mercaptopurine and azathioprine to reduce toxicity and improve efficacy in the management of IBD. Inflamm Bowel Dis 2015;21:445-52.

12 Stocco G, Cuzzoni E, De Iudicibus S, et al. Deletion of glutathione-s-transferase $\mathrm{m} 1$ reduces azathioprine metabolite concentrations in young patients with inflammatory bowel disease. J Clin Gastroenterol 2014;48:43-51.

13 Dubinsky MC. Azathioprine, 6-mercaptopurine in inflammatory bowel disease: pharmacology, efficacy, and safety. Clin Gastroenterol Hepatol 2004;2:731-43.

14 Ansari A, Aslam Z, De Sica A, et al. Influence of xanthine oxidase on thiopurine metabolism in Crohn's disease. Aliment Pharmacol Ther 2008;28:749-57.

15 Ansari A, Hassan C, Duley J, et al. Thiopurine methyltransferase activity and the use of azathioprine in inflammatory bowel disease. Aliment Pharmacol Ther 2002;16:1743-50.

16 Derijks LJ, Gilissen LP, Engels LG, et al. Pharmacokinetics of 6-thioguanine in patients with inflammatory bowel disease. Ther Drug Monit 2006;28:45-50.

17 Haglund S, Vikingsson S, Söderman J, et al. The role of inosine-5'-monophosphate dehydrogenase in thiopurine metabolism in patients with inflammatory bowel disease. Ther Drug Monit 2011;33:200-8.

18 Gisbert JP, Gomollón F. Thiopurine-induced myelotoxicity in patients with inflammatory bowel disease: a review. Am J Gastroenterol 2008;103:1783-800.

19 Cuffari C, Hunt S, Bayless T. Utilisation of erythrocyte 6-thioguanine metabolite levels to optimise azathioprine therapy in patients with inflammatory bowel disease. Gut 2001;48:642-6.

20 Thomas CW, Myhre GM, Tschumper R, et al. Selective inhibition of inflammatory gene expression in activated $\mathrm{T}$ lymphocytes: a mechanism of immune suppression by thiopurines. J Pharmacol Exp Ther 2005;312:537-45.

21 Tiede I, Fritz G, Strand S, et al. CD28-dependent Rac1 activation is the molecular target of azathioprine in primary human CD4+ T lymphocytes. J Clin Invest 2003;111:1133-45.

22 Hon YY, Fessing MY, Pui CH, et al. Polymorphism of the thiopurine S-methyltransferase gene in African-Americans. Hum Mol Genet 1999;8:371-6.

23 Colombel JF, Ferrari N, Debuysere H, et al. Genotypic analysis of thiopurine S-methyltransferase in patients with Crohn's 
disease and severe myelosuppression during azathioprine therapy. Gastroenterology 2000;118:1025-30.

24 Cuffari C, Dassopoulos T, Turnbough L, et al. Thiopurine methyltransferase activity influences clinical response to azathioprine in inflammatory bowel disease. Clin Gastroenterol Hepatol 2004;2:410-17.

25 Gisbert JP, Gomollón F, Cara C, et al. Thiopurine methyltransferase activity in Spain: a study of 14,545 patients. Dig Dis Sci 2007;52:1262-9.

26 González-Lama Y, Bermejo F, López-Sanromán A, et al. Thiopurine methyl-transferase activity and azathioprine metabolite concentrations do not predict clinical outcome in thiopurine-treated inflammatory bowel disease patients. Aliment Pharmacol Ther 2011;34:544-54.

27 Ansari A, Arenas M, Greenfield SM, et al. Prospective evaluation of the pharmacogenetics of azathioprine in the treatment of inflammatory bowel disease. Aliment Pharmacol Ther 2008;28:973-83.

28 Seinen ML, van Asseldonk DP, Mulder CJ, et al. Dosing 6-thioguanine in inflammatory bowel disease: expert-based guidelines for daily practice. J Gastrointestin Liver Dis 2010;19:291-4.

29 Dubinsky MC, Yang H, Hassard PV, et al. 6-MP metabolite profiles provide a biochemical explanation for 6-MP resistance in patients with inflammatory bowel disease. Gastroenterology 2002;122:904-15.

30 Pearson DC, May GR, Fick G, et al. Azathioprine for maintaining remission of Crohn's disease. Cochrane Database Syst Rev 2000;(2):Cd000067.

31 Present DH. 6-Mercaptopurine and other immunosuppressive agents in the treatment of Crohn's disease and ulcerative colitis. Gastroenterol Clin North Am 1989;18:57-71.

32 Bowen DG, Selby WS. Use of 6-mercaptopurine in patients with inflammatory bowel disease previously intolerant of azathioprine. Dig Dis Sci 2000;45:1810-13.

33 Nguyen TV, Vu DH, Nguyen TM, et al. Exploring associations of 6-thioguanine nucleotide levels and other predictive factors with therapeutic response to azathioprine in pediatric patients with IBD using multilevel analysis. Inflamm Bowel Dis 2013;19:2404-10.

34 Hindorf U, Andersson P. How are thiopurines used and monitored by Swedish gastroenterologists when treating patients with inflammatory bowel disease? Scand J Gastroenterol 2011;46:1215-21.

35 Roblin X, Oussalah A, Chevaux JB, et al. Use of thiopurine testing in the management of inflammatory bowel diseases in clinical practice: a worldwide survey of experts. Inflamm Bowel Dis 2011;17:2480-7.

36 Osterman MT, Kundu R, Lichtenstein GR, et al. Association of 6-thioguanine nucleotide levels and inflammatory bowel disease activity: a meta-analysis. Gastroenterology 2006;130:1047-53.

37 Gisbert JP, Gonzalez-Lama Y, Maté J. [Monitoring of thiopurine methyltransferase and thiopurine metabolites to optimize azathioprine therapy in inflammatory bowel disease]. Gastroenterol Hepatol 2006;29:568-83.

38 Angelberger S, Schaeffeler E, Teml A, et al. Mucosal improvement in patients with moderate to severe postoperative endoscopic recurrence of Crohn's disease and azathioprine metabolite levels. Inflamm Bowel Dis 2013;19:590-8.

39 Achkar JP, Stevens T, Easley K, et al. Indicators of clinical response to treatment with six-mercaptopurine or azathioprine in patients with inflammatory bowel disease. Inflamm Bowel Dis 2004;10:339-45.

40 Morales A, Salguti S, Miao CL, et al. Relationship between 6-mercaptopurine dose and 6-thioguanine nucleotide levels in patients with inflammatory bowel disease. Inflamm Bowel Dis 2007;13:380-5.

41 Lee MN, Kang B, Choi SY, et al. Relationship between azathioprine dosage, 6-thioguanine nucleotide levels, and therapeutic response in pediatric patients with IBD treated with azathioprine. Inflamm Bowel Dis 2015;21:1054-62.

42 Kennedy NA, Asser TL, Mountifield RE, et al. Thiopurine metabolite measurement leads to changes in management of inflammatory bowel disease. Intern Med J 2013;43:278-86.

43 Dassopoulos T, Dubinsky MC, Bentsen JL, et al. Randomised clinical trial: individualised vs. weight-based dosing of azathioprine in Crohn's disease. Aliment Pharmacol Ther 2014;39:163-75.

44 González Lama Y, Gisbert JP. Letter: metabolite monitoring for thiopurines in Crohn's disease-still not fully understood. Aliment Pharmacol Ther 2014;39:642.

45 Kopylov U, Battat R, Benmassaoud A, et al. Hematologic indices as surrogate markers for monitoring thiopurine therapy in IBD. Dig Dis Sci 2015;60:478-84.

46 Gisbert JP. Is less more: does leukopenia predict remission in patients with inflammatory bowel disease receiving thiopurine treatment? Dig Dis Sci 2015;60:4-6.

47 Heerasing NM, Ng JF, Dowling D. Does lymphopenia or macrocytosis reflect 6-thioguanine levels in patients with inflammatory bowel disease treated with azathioprine or 6-mercaptopurine? Intern Med J 1 Oct 2015. doi:10.1111/ imj.12915. [Epub ahead of print]

48 Dong XW, Zheng Q, Zhu MM, et al. Thiopurine $\mathrm{S}$-methyltransferase polymorphisms and thiopurine toxicity in treatment of inflammatory bowel disease. World J Gastroenterol 2010;16:3187-95.

49 Nguyen TV, Nguyen TM, Lachaux A, et al. Usefulness of thiopurine metabolites in predicting azathioprine resistance in pediatric IBD patients. J Clin Pharmacol 2013;53:900-8.

50 Kreijne JE, Seinen ML, Wilhelm AJ, et al. Routinely established skewed thiopurine metabolism leads to a strikingly high rate of early therapeutic failure in patients with inflammatory bowel disease. Ther Drug Monit 2015;37:797-804.

51 Gisbert JP, Gonzalez-Lama Y, Maté J. Thiopurine-induced liver injury in patients with inflammatory bowel disease: a systematic review. Am J Gastroenterol 2007;102:1518-27.

52 Dubinsky MC, Reyes E, Ofman J, et al. A cost-effectiveness analysis of alternative disease management strategies in patients with Crohn's disease treated with azathioprine or 6-mercaptopurine. Am J Gastroenterol 2005;100:2239-47.

53 Winter JW, Gaffney D, Shapiro D, et al. Assessment of thiopurine methyltransferase enzyme activity is superior to genotype in predicting myelosuppression following azathioprine therapy in patients with inflammatory bowel disease. Aliment Pharmacol Ther 2007;25:1069-77.

54 Roberts RL, Barclay ML. Current relevance of pharmacogenetics in immunomodulation treatment for Crohn's disease. J Gastroenterol Hepatol 2012;27:1546-54.

55 van Egmond R, Chin P, et al. High TPMT enzyme activity does not explain drug resistance due to preferential 6-methylmercaptopurine production in patients on thiopurine treatment. Aliment Pharmacol Ther 2012;35:1181-9.

56 Coenen MJ, de Jong DJ, van Marrewijk CJ, et al. Identification of Patients With Variants in TPMT and Dose 
Reduction Reduces Hematologic Events During Thiopurine Treatment of Inflammatory Bowel Disease. Gastroenterology 2015;149:907-17.e7.

57 Zabala W, Cruz R, Barreiro-de Acosta M, et al. New genetic associations in thiopurine-related bone marrow toxicity among inflammatory bowel disease patients. Pharmacogenomics 2013;14:631-40.

58 Lee MN, Woo HI, Lee YM, et al. Successful azathioprine treatment with metabolite monitoring in a pediatric inflammatory bowel disease patient homozygous for TPMT*3C. Yonsei Med J 2013;54:1545-9.

59 Gilissen LP, Wong DR, Engels LG, et al. Therapeutic drug monitoring of thiopurine metabolites in adult thiopurine tolerant IBD patients on maintenance therapy. J Crohns Colitis 2012;6:698-707.

60 Stocco G, Londero M, Campanozzi A, et al. Usefulness of the measurement of azathioprine metabolites in the assessment of non-adherence. J Crohns Colitis 2010;4:599-602.

61 Actis GC, Pellicano R, Rizzetto M, et al. Individually administered or co-prescribed thiopurines and mesalamines for inflammatory bowel disease. World J Gastroenterol 2009;15:1420-6.

62 Gao X, Zhang FB, Ding L, et al. The potential influence of 5 -aminosalicylic acid on the induction of myelotoxicity during thiopurine therapy in inflammatory bowel disease patients. Eur J Gastroenterol Hepatol 2012;24:958-64.

63 de Graaf P, de Boer NK, et al. Influence of 5-aminosalicylic acid on 6-thioguanosine phosphate metabolite levels: a prospective study in patients under steady thiopurine therapy. Br J Pharmacol 2010;160:1083-91.

64 Stocco G, Cuzzoni E, De Iudicibus S, et al. Thiopurine metabolites variations during co-treatment with aminosalicylates for inflammatory bowel disease: effect of $\mathrm{N}$-acetyl transferase polymorphisms. World J Gastroenterol 2015;21:3571-8.

65 Colombel JF, Sandborn WJ, Reinisch W, et al. Infliximab, azathioprine, or combination therapy for Crohn's disease. N Engl J Med 2010;362:1383-95.

66 Yarur AJ, Kubiliun MJ, Czul F, et al. Concentrations of 6-thioguanine nucleotide correlate with trough levels of infliximab in patients with inflammatory bowel disease on combination therapy. Clin Gastroenterol Hepatol 2015;13:1118-24.e3.

67 Van Assche G, Magdelaine-Beuzelin C, D’Haens G, et al. Withdrawal of immunosuppression in Crohn's disease treated with scheduled infliximab maintenance: a randomized trial. Gastroenterology 2008;134:1861-8.
68 Oussalah A, Chevaux JB, Fay R, et al. Predictors of infliximab failure after azathioprine withdrawal in Crohn's disease treated with combination therapy. Am J Gastroenterol 2010;105:1142-9.

69 Roblin X, Serre-Debeauvais F, Phelip JM, et al. 6-tioguanine monitoring in steroid-dependent patients with inflammatory bowel diseases receiving azathioprine. Aliment Pharmacol Ther 2005;21:829-39.

70 Teichgraber U, Atreya I, Atreya R, et al. Infliximab treatment induces levels of the active azathioprine metabolite TGTP in Crohn's disease. Inflamm Bowel Dis 2013;19:E54-5.

71 Wong DR, Pierik M, Seinen ML, et al. The pharmacokinetic effect of adalimumab on thiopurine metabolism in Crohn's disease patients. J Crohns Colitis 2014;8:120-8.

72 Shih DQ, Nguyen M, Zheng L, et al. Split-dose administration of thiopurine drugs: a novel and effective strategy for managing preferential 6-MMP metabolism. Aliment Pharmacol Ther 2012;36:449-58.

73 Appell ML, Wagner A, Hindorf U. A skewed thiopurine metabolism is a common clinical phenomenon that can be successfully managed with a combination of low-dose azathioprine and allopurinol. J Crohns Colitis 2013;7:510-13.

74 Ansari A, Patel N, Sanderson J, et al. Low-dose azathioprine or mercaptopurine in combination with allopurinol can bypass many adverse drug reactions in patients with inflammatory bowel disease. Aliment Pharmacol Ther 2010;31:640-7.

75 Sparrow MP, Hande SA, Friedman S, et al. Allopurinol safely and effectively optimizes tioguanine metabolites in inflammatory bowel disease patients not responding to azathioprine and mercaptopurine. Aliment Pharmacol Ther 2005;22:441-6.

76 Seinen ML, van Asseldonk DP, de Boer NK, et al. The effect of allopurinol and low-dose thiopurine combination therapy on the activity of three pivotal thiopurine metabolizing enzymes: results from a prospective pharmacological study. J Crohns Colitis 2013;7:812-19.

77 Blaker PA, Arenas-Hernandez M, Smith MA, et al. Mechanism of allopurinol induced TPMT inhibition. Biochem Pharmacol 2013;86:539-47.

78 Curkovic I, Rentsch KM, Frei P, et al. Low allopurinol doses are sufficient to optimize azathioprine therapy in inflammatory bowel disease patients with inadequate thiopurine metabolite concentrations. Eur J Clin Pharmacol 2013;69:1521-31.

79 Doré M, Frenette AJ, Mansour AM, et al. Febuxostat as a novel option to optimize thiopurines' metabolism in patients with inadequate metabolite levels. Ann Pharmacother 2014;48:648-51. 